\title{
A Critical Review of the Evidence Concerning the HIV Latency Reversing Effect of Disulfiram, the Possible Explanations for Its Inability to Reduce the Size of the Latent Reservoir In Vivo, and the Caveats Associated with Its Use in Practice
}

\author{
Harry D. J. Knights \\ Brasenose College, University of Oxford, Oxford, UK \\ Correspondence should be addressed to Harry D. J. Knights; harry.knights16@imperial.ac.uk \\ Received 10 January 2017; Revised 12 March 2017; Accepted 21 March 2017; Published 30 March 2017 \\ Academic Editor: Guido Poli \\ Copyright (C) 2017 Harry D. J. Knights. This is an open access article distributed under the Creative Commons Attribution License, \\ which permits unrestricted use, distribution, and reproduction in any medium, provided the original work is properly cited.

\begin{abstract}
Combination antiretroviral therapy (cART) effectively suppresses the replication of human immunodeficiency virus type 1 (HIV-1), improves immune function, and decreases the morbidity of acquired immune deficiency syndrome (AIDS). However, it is unable to eradicate the virus because it does not eliminate latently infected cells. The latent reservoir poses the major barrier to an HIV-1 cure. The "shock and kill" strategy aims to reactivate the virus and destroy latently infected cells. Many latency reversing agents (LRAs) reactivate HIV in vitro, but the absence of damaging side-effects and efficacy in vivo make disulfiram particularly promising. However, in clinical trials to date, disulfiram treatment has not resulted in a reduction in the size of the latent reservoir. In this article I will therefore discuss the evidence for the latency reversing effect of disulfiram, the possible explanations for its inability to reduce the size of the latent reservoir in vivo, and the caveats associated with its use in practice. These considerations will help to inform
\end{abstract} \\ judgements about the prospect of an HIV cure from disulfiram based treatments.
}

\section{Introduction}

Combination antiretroviral therapy (cART) for people infected with human immunodeficiency virus type 1 (HIV-1) effectively suppresses viral replication to below the limit of detection [1]. This successfully improves immune function and substantially decreases the morbidity and mortality of acquired immune deficiency syndrome (AIDS) [1]. However, it fails to eliminate the virus because a latent form of HIV persists $[2,3]$.

The latent reservoir consists of between $10^{5}$ and $10^{6}$ long-lived resting memory $\mathrm{CD}^{+}{ }^{+} \mathrm{T}$ cells $(\mathrm{Tm})$ harbouring an integrated, replication competent HIV genome [2-4]. Provided the host cell stays quiescent, the provirus remains transcriptionally silent [5]. This means that latently infected cells do not express viral antigen and therefore cannot be targeted by the immune system $[3,5]$. However, following cellular activation, the provirus is transcribed and virus is produced, reestablishing active infection $[2,3]$. The latent reservoir is extremely stable; it has been estimated to have a half-life of 44 months in HIV patients receiving cART, requiring 73.4 years to be completely destroyed [6]. This might be explained by the vertical transmission of infectious proviruses during the clonal expansion of Tm cells [7]. The recently discovered long-lived latently infected stem cell-like Tm cells (Tscm) [8] may also be involved. However, the significance of this rare population of cells remains unclear.

The stability of the latent reservoir means that HIV patients have to maintain cART for life. This is expensive, requires lifelong compliance, has undesirable side-effects, and creates stigma, providing the rational for the development of an HIV cure. Two types of cure are recognised: sterilising and functional. A sterilising cure would completely eradicate the virus (including latent virus), while a functional cure would result in long-term host-mediated control of viral replication and consequently lasting remission of symptoms in the absence of cART, with replication competent virus remaining within the body. 
The "shock and kill" [9] strategy has recently gained a lot of attention. This aims to reactivate the latent virus, reducing the size of the latent reservoir through direct viral cytopathic killing and immune-mediated destruction while cellular reinfection is blocked with cART [9]. Although this has traditionally been considered a sterilising cure strategy, recent studies on the natural hosts of Simian immunodeficiency viruses (SIVs) suggest that reduced central memory $\mathrm{CD} 4^{+}$ $\mathrm{T}$ cell $(\mathrm{Tcm})$ infection, in the absence of viral eradication, might be responsible for their functional cure $[10,11]$. It is therefore tempting to speculate that incomplete shock and kill strategies might reduce the size of the Tm pool enough for continued antiretroviral treatment to establish a functional cure in humans. Therefore, precisely what type of cure shock and kill is aiming to achieve is open to debate.

Early shock and kill attempts revolved around the use of immune activators: anti-CD3 immunoglobulin, IL-2, and TNF $\alpha[12,13]$. However, they entailed a high risk of global $\mathrm{T}$ cell activation and systemic induction of proinflammatory cytokines $[12,13]$. Therefore, latency reversing agents (LRAs) that activate HIV from latency without global T cell activation were needed. Since the turn of the century, an array of different compounds have shown promise in vitro [14-16]. Two of these, the histone deacetylase inhibitors (HDACI) [17-19] and disulfiram [20,21], have recently shown success in vivo. However, there have been indications in the literature of widespread and damaging HDACI side-effects [22], including disruption of the antiviral immune system [23], raising concerns about the feasibility of prolonged administration. Accordingly, in this article I will focus on disulfiram, although other LRAs will be discussed where appropriate.

1.1. Disulfiram. The latency reversal capabilities of disulfiram and its metabolites were first uncovered in 2011 by Xing et al. [15]. They established latency in $\mathrm{CD} 4^{+}$lymphoblasts by infecting them with HIV and then allowing them to return to a resting state. Elegantly, since they used recombinant HIV expressing GFP, they were able to assess reactivation by flow cytometry. Using this technique, they screened the John Hopkins Drug Library and found eight hits; one of them, disulfiram, had been used to safely treat alcoholism for decades under the trade-name "Antabuse" [24]. Crucially, disulfiram treatment did not increase cell size (an indicator of activity), the production of proinflammatory cytokines, or the expression of activation markers. Therefore, the latency reversal effect of disulfiram appeared to be HIV specific and not due to global $\mathrm{T}$ cell activation. The proposed mechanism is outlined in Figure 1.

The in vitro effect, however, did not translate into an effect on patient cells. In 2014, Bullen et al. examined the latency reversal effect of disulfiram on resting $\mathrm{CD} 4^{+} \mathrm{T}$ cells freshly isolated from HIV patients on cART [26]. Unexpectedly, they found that disulfiram did not induce viral outgrowth and virus release or cause increases in intracellular HIV mRNA. This suggested, in contrast to studies on in vitro models, that disulfiram does not reactivate latency in patient cells. The reasons for this discrepancy are unclear. One explanation is that current in vitro models for HIV latency do not fully recapitulate the mechanisms governing HIV latency in patient cells. Disappointingly, therefore, this study suggested

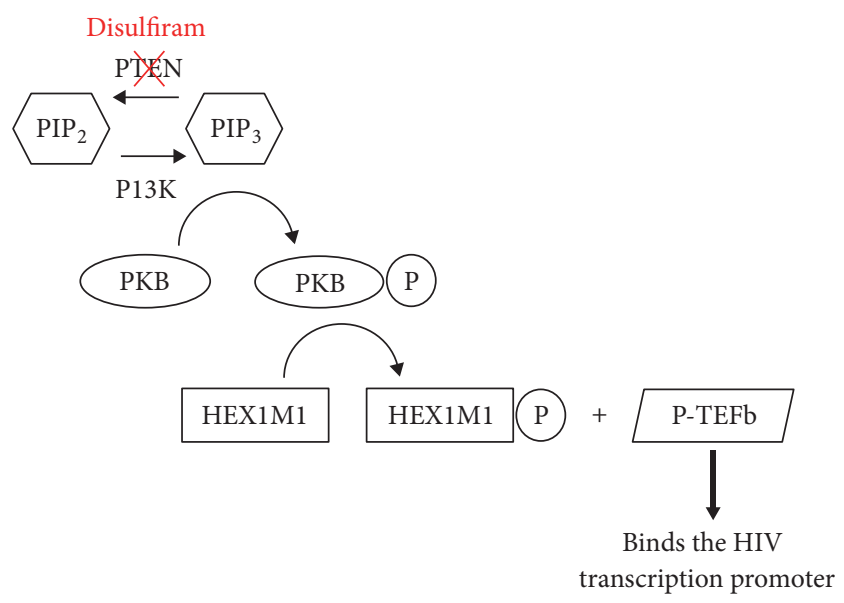

Figure 1: The mechanism of disulfiram activation of HIV transcription. Disulfiram activates the $\mathrm{PKB} /$ Akt signalling pathway by depleting PTEN (see Doyon et al. [25]).

that disulfiram would be unlikely to drive eradication of the latent reservoir in vivo.

At first, this was consistent with evidence from clinical trials. In 2014, Spivak et al. [20] administered $500 \mathrm{mg}$ of disulfiram daily for two weeks to HIV patients on stable cART. While cART is extremely effective at suppressing viral replication, patients on cART still develop a residual viraemia [27-29]. This is caused by the release of virus from latently infected cells following activation [28]. Therefore, the authors were able to determine latency reactivation in vivo by measuring plasma HIV RNA. In the original analysis, they found no significant change to plasma HIV RNA during disulfiram administration. However, in a post hoc analysis, a transient increase was detected in patients sampled immediately after dosing and patients with higher plasma disulfiram concentrations. These findings inspired a subsequent phase 2 dose-escalation study [21]. Patients received 500, 1000, or $2000 \mathrm{mg}$ of disulfiram daily for three days. A dose-related increase in cell-associated unspliced HIV RNA was observed during and after dosing. It remains unclear why Bullen et al. were unable to detect the same increase in intracellular HIV RNA ex vivo on patient cells. It is possible the immune system is having a synergistic effect on latency reactivation in vivo [26]. Collectively, these landmark clinical studies suggest that disulfiram provides a window of effective latency reactivation in vivo. This provides the first evidence of successful latency reversal in HIV infected individuals by a safe, well-tolerated drug and well-established drug.

\section{Discussion}

There are a number of problems with the use of disulfiram to eradicate the latent reservoir that need to be discussed.

2.1. The Persistence of the Latent Reservoir. Inconsistent with the shock and kill hypothesis, none of the disulfiram studies reported a decrease in the frequency of latently infected cells. 
Understanding why this is the case will be essential for the development of effective shock and kill cure strategies.

Disulfiram might simply not be potent enough to achieve the magnitude of latency reversal required to cause virusmediated cell lysis. If correct, it might be necessary to use disulfiram in combination with other mechanistically distinct LRAs to enhance the reactivating effect [30]. This might be compounded by the high frequency of viral escape mutations in patients on cART [31] and the impaired cytotoxic T cell (CTL) cytolytic capacity associated with chronic HIV infections [32], both predicted to inhibit the immune-mediated destruction of reactivated cells.

Interestingly, this is consistent with a study by Shan et al. showing that in vitro latency reversal using an HDACI, suberoylanilide hydroxamic acid (SAHA), did not result in the death of infected Tm cells despite viral cytopathic effects and the presence of autologous CTLs [33]. Instead, the efficient killing of infected cells required antigen-specific stimulation of patient CTLs prior to latency reactivation. It therefore appears that a balance between overstimulation, leading to global $\mathrm{T}$ cell activation, and understimulation, leading to the survival of reactivated cells, needs to be achieved. This has led to the general consensus that prestimulation of the antiviral immune response might be necessary for the successful "kill" of reactivated cells. Further studies will be required to confirm whether this applies to disulfiram in vivo.

2.2. The Presence of Other Viral Reservoirs. Many studies have examined the effect of disulfiram on latently infected Tm cells. However, less have investigated the effect of disulfiram on other cells. Many different cell types including macrophages [34], dendritic cells [35], and haematopoietic progenitor cells (HPCs) [36] are susceptible to HIV in vitro. Moreover, macrophages [34], microglia [37, 38], astrocytes [37-39], and HPCs [36] from HIV patients have been shown to contain HIV DNA. These findings have led to the proposition that latently infected Tm cells might not be the only viral reservoir in vivo. Whether or not this is the case is still far from certain. While other cell types can be infected in vivo and contain integrated HIV genomes, whether they persist for years in the setting of cART and can be reactivated to produce infectious virus is a contentious issue [40] (for a review, see Kandathil et al. 2016 [41]). Therefore, from a clinical perspective, it is unclear whether they are capable of reseeding infection.

Crucially, however, if non-Tm reservoirs do develop, they will be important to eradication efforts and will likely become more so once the Tm reservoir has been eliminated. Specifically, if disulfiram cannot target these reservoirs, cART could never be stopped for fear of viral rebound "restocking" the cleared reservoirs.

2.3. The Possibility of a CNS Reservoir. Recently, the possibility of a CNS reservoir has been the centre of attention [40]. This is because the CNS has a unique set of characteristics that could greatly affect the outcome of LRA use:

(1) The potential to develop HIV-associated neurocognitive disorders (HANDs) [42].
(2) Restricted LRA penetration [43] which may limit the "shock."

(3) Altered immune surveillance [44] which may compromise the "kill."

(4) Restricted cART penetration [45] which may allow continued viral replication, counteracting the clearance of the latent reservoir.

(5) Phylogenetically distinct HIV clades within the CNS which may respond differently to the LRA [46].

(6) Latently infected cells within the CNS (microglia and possibly astrocytes) are long-lived [47].

The development of HANDs is of particular importance. Owing to the success of cART, the life expectancy of HIV patients has increased dramatically over the last 20 years [48]. As a result, secondary HIV pathologies (such as HANDs) are becoming more frequent [40]. The pathophysiology underlying the development of HANDs is not known. However, one possibility is that they are caused by a long-lived infected CNS cell population that is releasing virus within the brains of suppressed patients. If this is the case, shock and kill strategies will need to target these cells. Conveniently, disulfiram easily crosses the blood-brain barrier because of its small size [49]. This means it is anatomically able to target any latently infected cells residing within the CNS. However, Gray et al. found it did not induce viral transcription in monocytederived macrophages, a cellular model for brain perivascular macrophages and microglia, or primary foetal astrocytes [50]. This result is disappointing: if reproducible in vivo, disulfiram may not improve the cognitive decline of HIV patients. Specific techniques for targeting CNS latency may therefore be needed in conjunction with disulfiram treatment.

However, the degree to which their cellular model represents latently infected CNS cells in vivo needs careful consideration. In particular, they validate their model by confirming the cell replication kinetics are equivalent to cultured microglia; this, however, is just one cell characteristic. They also used a lentiviral vector whose long-terminal repeat (LTR) was derived from the X4-tropic laboratory strain of HIV-1, NL4-3. The authors hypothesised that LTRs derived from CNS-tropic strains of HIV might respond differently to disulfiram. However, this is now known not to be the case. In a later study, the same group showed disulfiram did not activate transcription in human foetal astrocytic cells transfected with HIV containing LTRs from the CNS compartment of HIV patients [51]. Nevertheless, these findings need to be confirmed in animal models and then in vivo on HIV patients before any conclusions can be drawn about the efficacy of disulfiram on latently infected CNS cells.

On the other hand, if it transpires that disulfiram cannot target latently infected CNS cells, it is possible that this may not affect its therapeutic potential. Specifically, whether HANDs are caused by the release of virus from long-lived latently infected CNS cells remains unknown. Instead, it is possible that HANDs are a consequence of untreated viral infection prior to the initiation of cART or during periods of interrupted treatment. Moreover, only around $10 \%$ of HIV patients have detectable levels of HIV RNA in their 
cerebrospinal fluid (CSF) [52]. This might suggest that the presence of latently infected cells within the CNS is an uncommon occurrence. As a result, the precise implications of disulfiram being unable to reverse latency in CNS cells remain unclear.

Furthermore, it must also be considered that successful shock and kill within the CNS has the potential to cause cognitive decline itself through the destruction of infected glial cells and subsequent neuroinflammation. This would be a particular concern for patients with a larger number of latently infected cells within the CNS (perhaps because of late cART initiation or a long infection period). It might, therefore, be better to leave such patients on strict cART regimes. On the other hand, there may be situations where mild or transient neuronal dysfunction is an acceptable "price" for HIV eradication, similar to the "chemo brain" some patients experience following systemic cancer chemotherapy [53]. Either way, if LRAs like disulfiram were to be used clinically, neurocognitive monitoring would be essential to ensure any neurocognitive decline is detected in time for treatment to be stopped.

There is also the challenge of achieving adequate CNS penetration of antiretroviral agents to ensure the clearing of the CNS viral reservoir is not masked by continued CNS viral replication and propagation. The antiretroviral drug CNS penetration-effectiveness (CPE) rank [54] provides a useful guide and can be augmented by novel nanoformulation delivery techniques [55]. However, CNS penetration is potentially double-edged; in vitro studies suggest some antiretrovirals, particularly the NRTI abacavir and the NNRTI nevirapine, can be neurotoxic [56]. Therefore, while CNS cART infiltration will be essential for successful shock and kill, this problem should be approached with caution for fear of worsening any HANDs and impairing CNS function.

2.4. Measuring the Size of the Viral Reservoir. Parallel to the problem of eliminating the latent reservoir is the challenge of measuring the size of the latent pool to determine when the virus has been eradicated. This highlights another key problem facing efforts to eradicate HIV using disulfiram: techniques for measuring reservoir size are inaccurate and complicated. An in-depth analysis of the pros and cons of the different techniques used is beyond the scope of this article (for a review, see Bruner et al. [57]). However, in brief, there are two types: quantitative viral outgrowth assays (Q-VOA) and polymerase chain reaction- (PCR-) based methods.

The Q-VOA uses phytohemagglutinin to globally activate Tm cells ex vivo and reinstate HIV production. The virus is then expanded and quantified by ELISA for the HIV p24 antigen. While this approach has long been the "gold standard," a recent study identified intact proviruses from p24 negative samples [58], suggesting that the Q-VOA underestimates the size of the in vivo reservoirs. This is believed to be because reactivation is stochastic, such that each provirus has a finite probability of being induced following activation [59]. Furthermore, the Q-VOA is labour intensive and expensive, has a 1-3 week culture time, and requires large blood volumes. Accordingly, it remains unsuitable for large scale clinical trials.
Alternatively, the PCR methods directly measure the frequency of cells containing HIV genomes [60]. Here, PCR is performed on blood Tm cell DNA and the infected cell frequency is calculated by comparison to a standard curve constructed using known copy numbers of proviral DNA. Although PCR methods are simple and require small blood volumes, they quantify large numbers of dysfunctional, replication incompetent viral genomes [61]. In fact, PCR methods typically yield infected cell frequencies 2-3 logs higher than the Q-VOA [61]. Generally, therefore, the Q-VOA provides an underestimate and PCR an overestimate, of the true size of the latent pool. Using this information, it would be useful to create an equation to more precisely calculate true reservoir size from the Q-VOA and PCR estimates. This could be done in vitro by controlling latent reservoir size, measuring it with the Q-VOA and PCR methods, and then comparing the values.

Furthermore, nonspecificity means it is very difficult for PCR methods to detect small changes in the number of intact proviruses capable of reseeding infection. This is problematic because very few latently infected cells contain an intact provirus [62], and therefore successful shock and kill will only result in a small absolute reduction in the number of intact proviruses. Therefore, it is possible that the disulfiram studies underestimated the latency reversing effect on replication competent provirus.

To this end, perhaps the best technique to date has been the tat/rev induced limiting dilution assay (TILDA) [63]. This measures the frequency of cells containing inducible multiply spliced tat/rev RNA. This is usually absent from latent cells and is only induced upon reactivation [64]. Conversely, many defective genomes have deletions encompassing the tat and rev genes [58]. As a result, the latent cell frequency measured by TILDA lies somewhere between the Q-VOA and PCR estimates, indicative of greater accuracy. There are also no RNA extraction or virus amplification steps, meaning it takes just two days, and it offers similar advantages to PCR with regard to simplicity and small blood volume requirement. This makes TILDA a potentially useful assay for large scale clinical trials. However, again there are limitations: it relies on the amplification of a highly variable HIV genome region, suggesting that the required primers may not recognise all viral quasispecies.

Furthermore, as previously discussed, other latent cell types exist within tissues. This would not be a hindrance if the size of blood and tissue reservoirs was tightly correlated, but there is conflicting and limited evidence for this [65]. It might therefore be necessary to combine these quantification methods with tissue sampling techniques. This has been done previously [66]. However, biopsies pose their own limitations: they are invasive and cannot be taken from all tissues, especially the CNS. For the CNS, it may be possible to estimate the number of latently infected cells from the CSF viral load. However, further studies will be needed to confirm whether CSF viral load is dependent on the frequency of latently infected cells that may be residing within the CNS.

As can be seen, all techniques for quantifying the reservoir have their own advantages and disadvantages. This poses a serious problem for HIV eradication efforts. Without a 
high-throughput, sensitive, and well-validated assay it will remain difficult for researchers to move forward with clinical trials, accurately evaluate the efficacy of LRAs like disulfiram, and ever confirm that a sterilising cure has been achieved.

\section{Conclusions}

Overall, there is strong evidence that disulfiram effectively and specifically reverses HIV latency in cellular models. While this failed to translate into an effect on patient cells, subsequent clinical trials identified an effect in vivo. However, so far, this has failed to result in a reduction in the size of the latent reservoir in HIV patients. Possible explanations for this include insufficient potency and failed immune clearance. Therefore, it is likely that combining disulfiram with other mechanistically distinct LRAs and the use of preimmune stimulation will help overcome these challenges. Furthermore, future studies will be needed to assess whether non-Tm reservoirs are able to reseed the infection after prolonged cART. If they are, safe shock and kill techniques for nonblood reservoirs will need to be explored. Finally, more refined techniques for measuring the size of the latent reservoir safely in different tissues need to be developed. Therefore, while disulfiram shows promise as an HIV cure strategy, further studies are needed to solve the problems highlighted in this article before any conclusions about its clinical efficacy can be drawn.

\section{Conflicts of Interest}

The author has no conflicts of interest.

\section{Acknowledgments}

The author acknowledges Professor William James for his assistance.

\section{References}

[1] F. J. Palella Jr., K. M. Delaney, A. C. Moorman et al., "Declining morbidity and mortality among patients with advanced human immunodeficiency virus infection. HIV outpatient study investigators," The New England Journal of Medicine, vol. 338, no. 13, pp. 853-860, 1998.

[2] D. Finzi, M. Hermankova, T. Pierson et al., "Identification of a reservoir for HIV-1 in patients on highly active antiretroviral therapy," Science, vol. 278, no. 5341, pp. 1295-1300, 1997.

[3] J. K. Wong, M. Hezareh, H. F. Günthard et al., "Recovery of replication-competent HIV despite prolonged suppression of plasma viremia," Science, vol. 278, no. 5341, pp. 1291-1295, 1997.

[4] T.-W. Chun, L. Carruth, D. Finzi et al., "Quantification of latent tissue reservoirs and total body viral load in HIV-1 infection," Nature, vol. 387, no. 6629, pp. 183-188, 1997.

[5] M. Hermankova, J. D. Siliciano, Y. Zhou et al., "Analysis of human immunodeficiency virus type 1 gene expression in latently infected resting $\mathrm{CD} 4^{+} \mathrm{T}$ lymphocytes in vivo," Journal of Virology, vol. 77, no. 13, pp. 7383-7392, 2003.

[6] J. D. Siliciano, J. Kajdas, D. Finzi et al., "Long-term follow-up studies confirm the stability of the latent reservoir for HIV-1 in resting CD4+ T cells," Nature Medicine, vol. 9, no. 6, pp. 727$728,2003$.

[7] F. R. Simonetti, M. D. Sobolewski, E. Fyne et al., "Clonally expanded $\mathrm{CD}^{+} \mathrm{T}$ cells can produce infectious HIV-1 in vivo," Proceedings of the National Academy of Sciences of the United States of America, vol. 113, no. 7, pp. 1883-1888, 2016.

[8] M. J. Buzon, H. Sun, C. Li et al., "HIV-1 persistence in CD $4^{+}$ T cells with stem cell-like properties," Nature Medicine, vol. 20, no. 2, pp. 139-142, 2014.

[9] G. Lehrman, I. B. Hogue, S. Palmer et al., "Depletion of latent HIV-1 infection in vivo: a proof-of-concept study," The Lancet, vol. 366, no. 9485, pp. 549-555, 2005.

[10] C. M. Beaumier, L. D. Harris, S. Goldstein et al., "CD4 downregulation by memory CD4+ T cells in vivo renders African green monkeys resistant to progressive SIVagm infection," Nature Medicine, vol. 15, no. 8, pp. 879-885, 2009.

[11] M. Paiardini, B. Cervasi, E. Reyes-Aviles et al., "Low levels of SIV infection in sooty mangabey central memory $\mathrm{CD} 4^{+} \mathrm{T}$ cells are associated with limited CCR5 expression," Nature Medicine, vol. 17, no. 7, pp. 830-836, 2011.

[12] T.-W. Chun, D. Engel, S. B. Mizell et al., "Effect of interleukin-2 on the pool of latently infected, resting CD4+ T cells in HIV-1infected patients receiving highly active anti-retroviral therapy," Nature Medicine, vol. 5, no. 6, pp. 651-655, 1999.

[13] J. M. Prins, S. Jurriaans, R. M. E. Van Praag et al., "Immunoactivation with anti-CD3 and recombinant human IL-2 in HIV1-infected patients on potent antiretroviral therapy," AIDS, vol. 13, no. 17, pp. 2405-2410, 1999.

[14] X. Contreras, M. Schweneker, C.-S. Chen et al., "Suberoylanilide hydroxamic acid reactivates HIV from latently infected cells," The Journal of Biological Chemistry, vol. 284, no. 11, pp. 67826789, 2009.

[15] S. Xing, C. K. Bullen, N. S. Shroff et al., "Disulfiram reactivates latent $\mathrm{HIV}-1$ in a Bcl-2-transduced primary $\mathrm{CD} 4^{+} \mathrm{T}$ cell model without inducing global T cell activation," Journal of Virology, vol. 85, no. 12, pp. 6060-6064, 2011.

[16] L. Ylisastigui, N. M. Archin, G. Lehrman, R. J. Bosch, and D. M. Margolis, "Coaxing HIV-1 from resting CD4 T cells: histone deacetylase inhibition allows latent viral expression," AIDS, vol. 18, no. 8, pp. 1101-1108, 2004.

[17] N. M. Archin, A. L. Liberty, A. D. Kashuba et al., "Administration of vorinostat disrupts HIV-1 latency in patients on antiretroviral therapy," Nature, vol. 487, no. 7408, pp. 482-485, 2012.

[18] J. H. Elliott, F. Wightman, A. Solomon et al., "Activation of HIV transcription with short-course vorinostat in HIV-infected patients on suppressive antiretroviral therapy," PLoS Pathogens, vol. 10, no. 11, Article ID e1004473, 2014.

[19] T. A. Rasmussen, M. Tolstrup, C. R. Brinkmann et al., "Panobinostat, a histone deacetylase inhibitor, for latent-virus reactivation in HIV-infected patients on suppressive antiretroviral therapy: a phase $1 / 2$, single group, clinical trial," The Lancet $H I V$, vol. 1, no. 1, pp. e13-e21, 2014.

[20] A. M. Spivak, A. Andrade, E. Eisele et al., "A pilot study assessing the safety and latency-reversing activity of disulfiram in HIV1-infected adults on antiretroviral therapy," Clinical Infectious Diseases, vol. 58, no. 6, pp. 883-890, 2014.

[21] J. H. Elliott, J. H. McMahon, C. C. Chang et al., "Short-term administration of disulfiram for reversal of latent HIV infection: a phase 2 dose-escalation study," The Lancet HIV, vol. 2, no. 12, pp. e520-e529, 2015. 
[22] S. Subramanian, S. E. Bates, J. J. Wright, I. Espinoza-Delgado, and R. L. Piekarz, "Clinical toxicities of histone deacetylase inhibitors," Pharmaceuticals, vol. 3, no. 9, pp. 2751-2767, 2010.

[23] R. B. Jones, R. O'Connor, S. Mueller et al., "Histone deacetylase inhibitors impair the elimination of HIV-infected cells by cytotoxic T-lymphocytes," PLoS Pathogens, vol. 10, no. 8, Article ID e1004287, 2014.

[24] R. G. Bell and H. W. Smith, "Preliminary report on clinical trials of antabuse," Canadian Medical Association Journal, vol. 60, no. 3, pp. 286-288, 1949.

[25] G. Doyon, J. Zerbato, J. W. Mellors, and N. Sluis-Cremer, “Disulfiram reactivates latent HIV-1 expression through depletion of the phosphatase and tensin homolog," AIDS, vol. 27, no. 2, pp. F7-F11, 2013.

[26] C. K. Bullen, G. M. Laird, C. M. Durand, J. D. Siliciano, and R. F. Siliciano, "New ex vivo approaches distinguish effective and ineffective single agents for reversing HIV-1 latency in vivo," Nature Medicine, vol. 20, no. 4, pp. 425-429, 2014.

[27] G. Dornadula, H. Zhang, B. VanUitert et al., "Residual HIV1 RNA in blood plasma of patients taking suppressive highly active antiretroviral therapy," The Journal of the American Medical Association, vol. 282, no. 17, pp. 1627-1632, 1999.

[28] F. Maldarelli, S. Palmer, M. S. King et al., "ART suppresses plasma HIV-1 RNA to a stable set point predicted by pretherapy viremia," PLoS Pathogens, vol. 3, no. 4, p. e46, 2007.

[29] S. Palmer, F. Maldarelli, A. Wiegand et al., "Low-level viremia persists for at least 7 years in patients on suppressive antiretroviral therapy," Proceedings of the National Academy of Sciences of the United States of America, vol. 105, no. 10, pp. 3879-3884, 2008.

[30] G. M. Laird, C. K. Bullen, D. I. S. Rosenbloom et al., "Ex vivo analysis identifies effective HIV-1 latency-reversing drug combinations," The Journal of Clinical Investigation, vol. 125, no. 5, pp. 1901-1912, 2015.

[31] K. Deng, M. Pertea, A. Rongvaux et al., "Broad CTL response is required to clear latent HIV-1 due to dominance of escape mutations," Nature, vol. 517, no. 7534, pp. 381-385, 2015.

[32] A. Sáez-Cirión, C. Lacabaratz, O. Lambotte et al., "HIV controllers exhibit potent CD8 T cell capacity to suppress HIV infection ex vivo and peculiar cytotoxic T lymphocyte activation phenotype," Proceedings of the National Academy of Sciences of the United States of America, vol. 104, no. 16, pp. 6776-6781, 2007.

[33] L. Shan, K. Deng, N. S. Shroff et al., "Stimulation of HIV-1specific cytolytic T lymphocytes facilitates elimination of latent viral reservoir after virus reactivation," Immunity, vol. 36, no. 3 , pp. 491-501, 2012.

[34] S. Gartner, P. Markovits, D. M. Markovitz, M. H. Kaplan, R. C. Gallo, and M. Popovic, "The role of mononuclear phagocytes in HTLV-III/LAV infection," Science, vol. 233, no. 4760, pp. 215219, 1986.

[35] S. Popov, A.-L. Chenine, A. Gruber, P.-L. Li, and R. M. Ruprecht, "Long-term productive human immunodeficiency virus infection of CDla-sorted myeloid dendritic cells," Journal of Virology, vol. 79, no. 1, pp. 602-608, 2005.

[36] C. C. Carter, A. Onafuwa-Nuga, L. A. McNamara et al., "HIV1 infects multipotent progenitor cells causing cell death and establishing latent cellular reservoirs," Nature Medicine, vol. 16, no. 4, pp. 446-451, 2010.

[37] M. J. Churchill, P. R. Gorry, D. Cowley et al., "Use of laser capture microdissection to detect integrated HIV-1 DNA in macrophages and astrocytes from autopsy brain tissues," Journal of NeuroVirology, vol. 12, no. 2, pp. 146-152, 2006.

[38] K. A. Thompson, C. L. Cherry, J. E. Bell, and C. A. McLean, "Brain cell reservoirs of latent virus in presymptomatic HIVinfected individuals," American Journal of Pathology, vol. 179, no. 4, pp. 1623-1629, 2011.

[39] C. A. Wiley, R. D. Schrier, J. A. Nelson, P. W. Lampert, and M. B. Oldstone, "Cellular localization of human immunodeficiency virus infection within the brains of acquired immune deficiency syndrome patients," Proceedings of the National Academy of Sciences of the United States of America, vol. 83, no. 18, pp. 70897093, 1986.

[40] B. J. Brew, K. Robertson, E. J. Wright et al., "HIV eradication symposium: will the brain be left behind?" Journal of NeuroVirology, vol. 21, no. 3, pp. 322-334, 2015.

[41] A. J. Kandathil, S. Sugawara, and A. Balagopal, "Are T cells the only HIV-1 reservoir?” Retrovirology, vol. 13, no. 1, p. 86, 2016.

[42] R. K. Heaton, D. B. Clifford, D. R. Franklin et al., "HIV-associated neurocognitive disorders persist in the era of potent antiretroviral therapy: CHARTER Study," Neurology, vol. 75, no. 23, pp. 2087-2096, 2010.

[43] M. J. Churchill, D. J. Cowley, S. L. Wesselingh, P. R. Gorry, and L. R. Gray, "HIV-1 transcriptional regulation in the central nervous system and implications for HIV cure research," Journal of NeuroVirology, vol. 21, no. 3, pp. 290-300, 2015.

[44] M. J. Carson, J. M. Doose, B. Melchior, C. D. Schmid, and C. C. Ploix, "CNS immune privilege: hiding in plain sight," Immunological Reviews, vol. 213, no. 1, pp. 48-65, 2006.

[45] S. Letendre, J. Marquie-Beck, E. Capparelli et al., "Validation of the CNS penetration-effectiveness rank for quantifying antiretroviral penetration into the central nervous system," Archives of Neurology, vol. 65, no. 1, pp. 65-70, 2008.

[46] M. Ait-Khaled, J. E. McLaughlin, M. A. Johnson, and V. C. Emery, "Distinct HIV-1 long terminal repeat quasispecies present in nervous tissues compared to that in lung, blood and lymphoid tissues of an AIDS patient," AIDS, vol. 9, no. 7, pp. 675-683, 1995.

[47] S. Kramer-Hämmerle, I. Rothenaigner, H. Wolff, J. E. Bell, and R. Brack-Werner, "Cells of the central nervous system as targets and reservoirs of the human immunodeficiency virus," Virus Research, vol. 111, no. 2, pp. 194-213, 2005.

[48] H. Samji, A. Cescon, R. S. Hogg et al., "Closing the gap: increases in life expectancy among treated HIV-positive individuals in the United States and Canada," PLoS ONE, vol. 8, no. 12, Article ID e81355, 2013.

[49] D. I. Eneanya, J. R. Bianchine, D. O. Duran, and B. D. Andresen, "The actions of metabolic fate of disulfiram," Annual Review of Pharmacology and Toxicology, vol. 21, pp. 575-596, 1981.

[50] L. R. Gray, H. On, E. Roberts et al., "Toxicity and in vitro activity of HIV-1 latency-reversing agents in primary CNS cells," Journal of NeuroVirology, vol. 22, no. 4, pp. 455-463, 2016.

[51] L. R. Gray, D. Cowley, C. Welsh et al., "CNS-specific regulatory elements in brain-derived HIV-1 strains affect responses to latency-reversing agents with implications for cure strategies," Molecular Psychiatry, vol. 21, no. 4, pp. 574-584, 2016.

[52] A. Edén, D. Fuchs, L. Hagberg et al., "HIV-1 viral escape in cerebrospinal fluid of subjects on suppressive antiretroviral treatment," Journal of Infectious Diseases, vol. 202, no. 12, pp. 18191825, 2010.

[53] C. B. Brezden, K.-A. Phillips, M. Abdolell, T. Bunston, and I. F. Tannock, "Cognitive function in breast cancer patients 
receiving adjuvant chemotherapy," Journal of Clinical Oncology, vol. 18, no. 14, pp. 2695-2701, 2000.

[54] S. Letendre, "Central nervous system complications in HIV disease: HIV-associated neurocognitive disorder," Topics in Antiviral Medicine, vol. 19, no. 4, pp. 137-142, 2011.

[55] H. Dou, C. B. Grotepas, J. M. McMillan et al., "Macrophage delivery of nanoformulated antiretroviral drug to the brain in a murine model of NeuroAIDS," Journal of Immunology, vol. 183, no. 1, pp. 661-669, 2009.

[56] K. Robertson, J. Liner, and R. B. Meeker, "Antiretroviral neurotoxicity, Journal of NeuroVirology, vol. 18, no. 5, pp. 388-399, 2012.

[57] K. M. Bruner, N. N. Hosmane, and R. F. Siliciano, "Towards an HIV-1 cure: measuring the latent reservoir," Trends in Microbiology, vol. 23, no. 4, pp. 192-203, 2015.

[58] Y.-C. Ho, L. Shan, N. N. Hosmane et al., "Replication-competent noninduced proviruses in the latent reservoir increase barrier to HIV-1 cure," Cell, vol. 155, no. 3, pp. X540-551, 2013.

[59] A. D. Weinberger and L. S. Weinberger, "Stochastic fate selection in HIV-infected patients," Cell, vol. 155, no. 3, pp. 497-499, 2013.

[60] C. Vandergeeten, R. Fromentin, E. Merlini et al., "Cross-clade ultrasensitive PCR-based assays to measure HIV persistence in large-cohort studies," Journal of Virology, vol. 88, no. 21, pp. 12385-12396, 2014.

[61] S. Eriksson, E. H. Graf, V. Dahl et al., "Comparative analysis of measures of viral reservoirs in HIV-1 eradication studies," PLoS Pathogens, vol. 9, no. 2, Article ID e1003174, 2013.

[62] K. M. Bruner, A. J. Murray, R. A. Pollack et al., "Defective proviruses rapidly accumulate during acute HIV-1 infection," Nature Medicine, vol. 22, no. 9, pp. 1043-1049, 2016.

[63] F. A. Procopio, R. Fromentin, D. A. Kulpa et al., "A novel assay to measure the magnitude of the inducible viral reservoir in HIVinfected individuals," EBioMedicine, vol. 2, no. 8, pp. 874-883, 2015.

[64] S. R. Lewin, M. Vesanen, L. Kostrikis et al., "Use of real-time PCR and molecular beacons to detect virus replication in human immunodeficiency virus type 1-infected individuals on prolonged effective antiretroviral therapy," Journal of Virology, vol. 73, no. 7, pp. 6099-6103, 1999.

[65] G. Pantaleo, C. Graziosi, J. F. Demarest et al., "HIV infection is active and progressive in lymphoid tissue during the clinically latent stage of disease," Nature, vol. 362, no. 6418, pp. 355-358, 1993.

[66] R. D. Hockett, J. M. Kilby, C. A. Derdeyn et al., "Constant mean viral copy number per infected cell in tissues regardless of high, low, or undetectable plasma HIV RNA," Journal of Experimental Medicine, vol. 189, no. 10, pp. 1545-1554, 1999. 


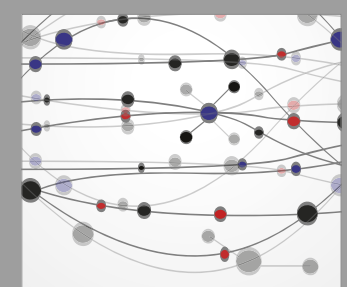

The Scientific World Journal
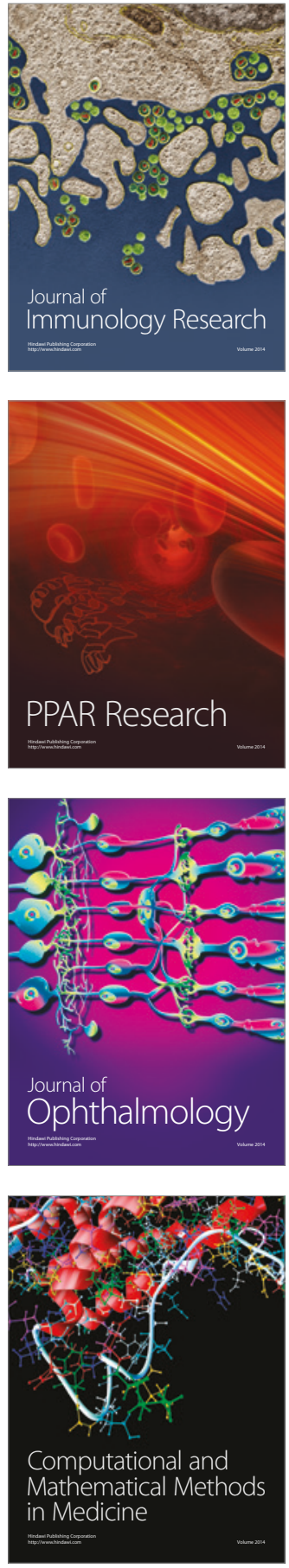

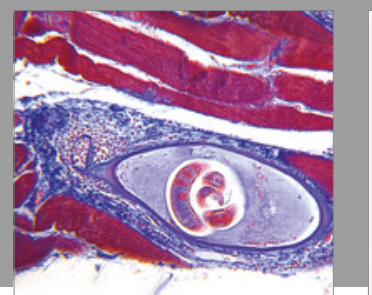

Gastroenterology Research and Practice
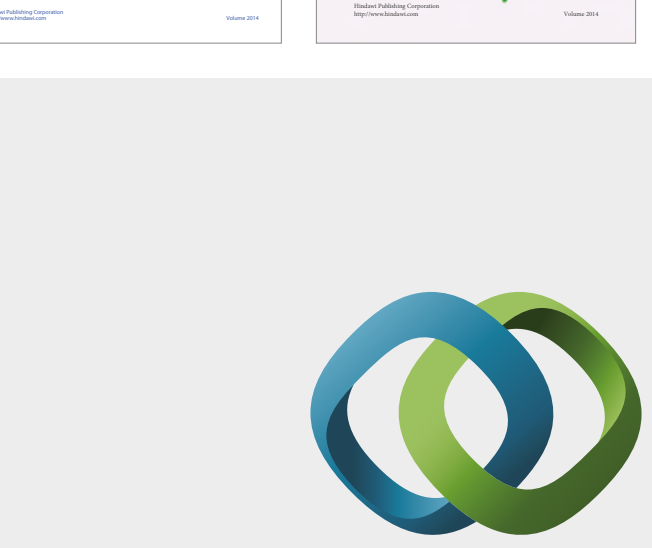

\section{Hindawi}

Submit your manuscripts at

https://www.hindawi.com
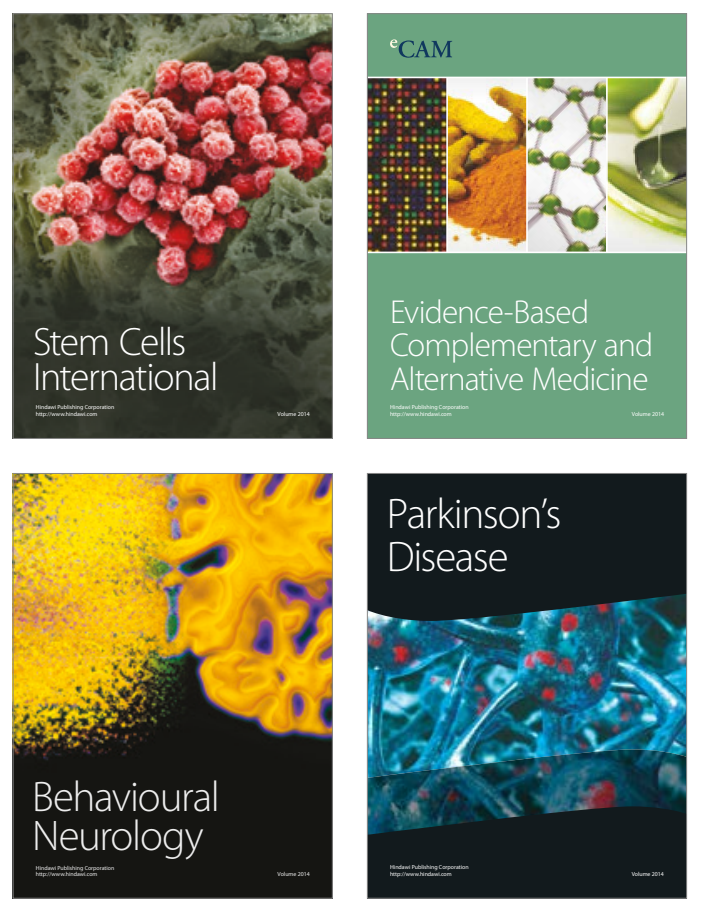
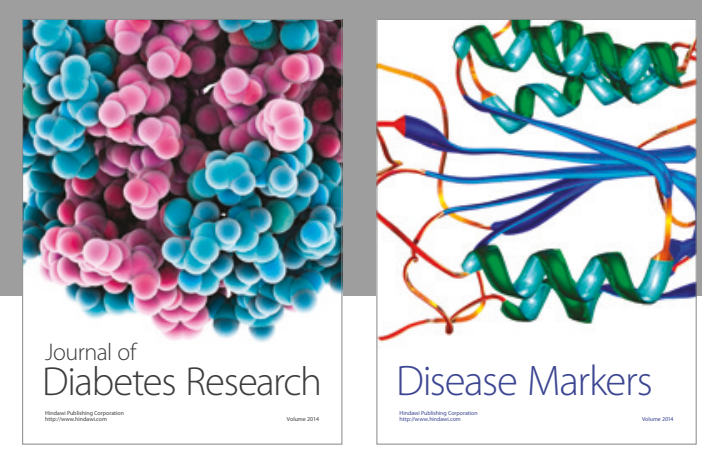

Disease Markers
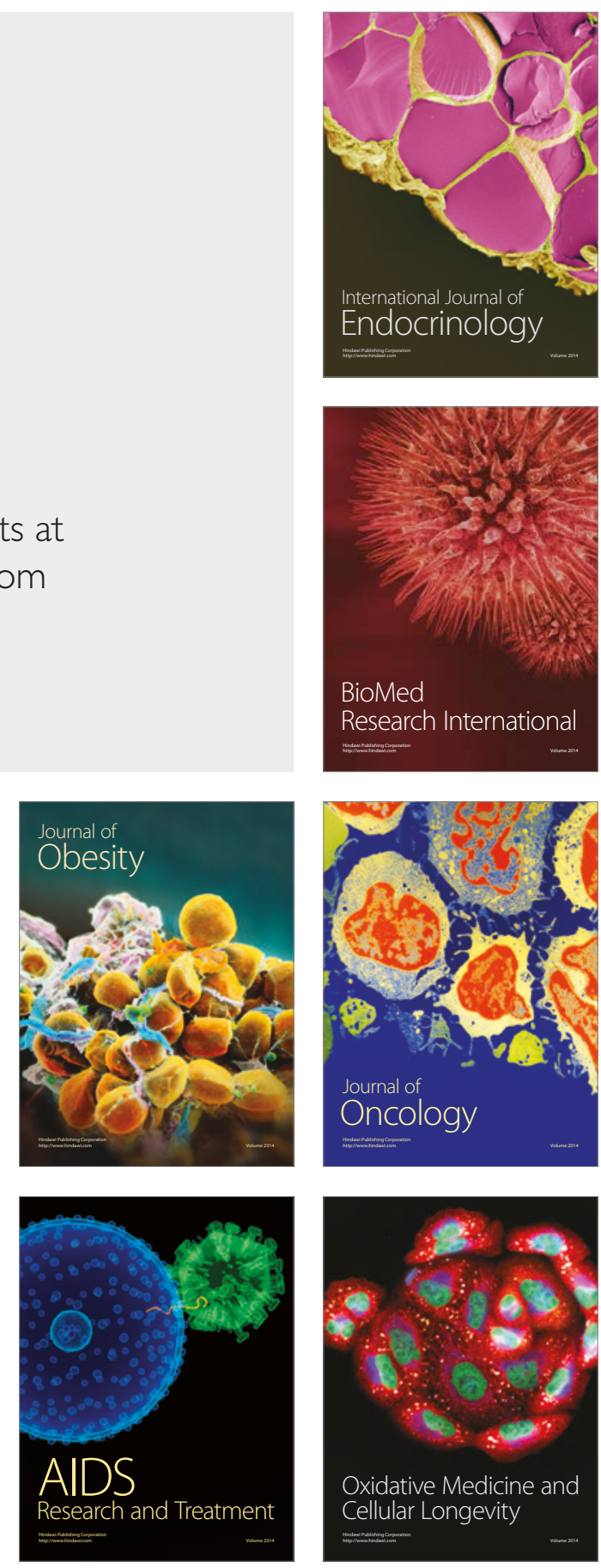